\title{
The Kanehira-Hatusima 1940 Collection of New Guinea Plants. XVI.
}

\author{
$\mathrm{By}$ \\ J. Ohwi. \\ Received August 4, 1942.

\section{J. Oнwі: Melastomaceae.} \\ 1. Otanthera novoguineensis BAKER fil. in RIDL. in Trans. Linn. Soc. \\ 2 ser. Bot. $9: 1$ (1916) 50 et in GibBs, Contrib. F. Phytog. Arfak Mts. (1917) \\ 216 ; Mansf. in Engl. Bot. Jahrb. 60 (1926) 107. \\ No. 11702 Kanehira-Hatusima, Nabire, Geelvink Bay, fairly common \\ in rain-forests, at about $50 \mathrm{~m}$. alt., Febr. 27, 1940. A shrub about $1 \mathrm{~m}$. tall. \\ No. 11627 Kanehira-Hatusima, Papaya, Nabire, fairly common in rain- \\ forests, at about $100 \mathrm{~m}$. alt., Febr. 27, 1940. A shrub about $1 \mathrm{~m}$. tall. \\ Distr. Endemic.
}

2. Melastoma patulisetum OHwI, sp. nov.

Frutex ramosus erectus. Rami paleis patentibus dense vestiti teretes. Folia oblongo-ovata tenuiter chartacea $\mathbf{1 0 - 1 5} \mathrm{cm}$. longa $4-5 \mathrm{~cm}$. lata pallide viridia, supra dense molliterque setulosa, subtus molliter pilosa, nervis subtus adpresse paleaceo-setulosa, utrinque acuta vel apice breviter acuminata, 5-plinervia, nervulis transversalibus tenuissimis, petiolis ascendentisubulato-paleaceis 15-20 mm. longis. Inflorescentia pauciflora sessilis $2 \mathrm{~cm}$. longa, pedicellis erectis $6-7 \mathrm{~mm}$. longis, juventute basi bracteis 2 spathulatooblongis $6-7 \mathrm{~mm}$. longis facile deciduis stipatis, paleis rubescentibus $1.5-2$ $\mathrm{mm}$. longis adpressis subulato-linearibus compressis acuminatis margine parce ciliolatis dense obtectis. Flores 5 -meri, calycis tubo $5 \mathrm{~mm}$. longo et lato paleis adpressis subulato-linearibus acuminatis rubescentibus $1.5-2 \mathrm{~mm}$. longis margine parce ciliolatis (vide fig. $\mathrm{B}$ ) dense vestito, lobis subulatolanceolatis acuminatis $3 \mathrm{~mm}$. longis deciduis, petalis roseis (ex coll.). Stamina 10 inaequalia, filamentis ca. $4 \mathrm{~mm}$. longis, antheris majoribus $4 \mathrm{~mm}$. longis (parte producta 5-6 mm. longa excepta) basi antice bilobis, antheris minoribus basi non producta bituberculatis, stylo $10 \mathrm{~mm}$. longo glabro. A M. Roemeri Mansf., cui proxime affine, differt calyce pedicellisque paleis longioribus acuminatis saepe rubescentibus vestitis, connectivo antherae majoris basi longe producto.

No. 13180 Kanehira-Hatusima, Waren, 60 miles south of Manokwari, 
in open rain-forests on a lime-stone mountain at about $500 \mathrm{~m}$. high, March 26, 1940. A shrub about $2 \mathrm{~m}$. tall, flowers pink.

3. Melastoma Roemeri Mansf. in EngL. Bot. Jahrb. 60 (1926) 108 nom. nud. et in Nova Guinea, 14 (1927) 200.

Nos. 11815, 11855 Kanehira-Hatusima, Chaban, Nabire, in rain-forests, alt. about 200-300 m. above the sea, Febr. 28, 1940. A shrub about $2 \mathrm{~m}$. tall, flowers pink.

Distr. Endemic.

\section{Poikilogyne mucronato-serrulata OHwi, sp. nov.}

$1.5 \mathrm{~m}$. alta (ex coll.) glaberrima. Rami acute tetraquetri $3-4 \mathrm{~mm}$. diametientes elongati. Folia opposita aequalia ovata vel lata ovata cum petiolo (rubescente $15-20 \mathrm{~mm}$. longo) $9-10 \mathrm{~cm}$. longa $3.5-5 \mathrm{~cm}$. lata subcoriacea viridia opaca 5-plinervia, margine minute mucronato-serrulata, apice acuta, basi rotundata vel subcordata, nervis supra leviter impressis, subtus cum nervis transversalibus rubescentibus elevatis. Panicula terminalis pyramidata, pedunculo $5 \mathrm{~cm}$. longo excepto, $10 \mathrm{~cm}$. longa et lata laxe multiflora, ramis divaricatis teretibus divaricato-ramosis, bracteis miminis, pedicellis 7-20 mm. longis supra medium saepe minutissime bibracteolatis. Flores 5-meri, rosei (ex coll.), calyce campanulato glabro $5 \mathrm{~mm}$. longo sub limbo leviter constricto, limbo $1.5 \mathrm{~mm}$. longo dilatato truncato- 5 -undulato, dentibus vix evolutis, petalis 5 obovato-ellipticis $8 \mathrm{~mm}$. longis subtruncatis vel leviter retusis 1-mucronatis, dorso superne margineque obsolete papulosis. Stamina 10 aequalia flavescentia, filamentis $3 \mathrm{~mm}$. longis, antheris lanceolatis obtusis, excepto appendiculo $3 \mathrm{~mm}$. longis, basi antice rotundatis inappendiculatis, postice tuberculo 1 carnoso vix $1 \mathrm{~mm}$. longo elliptico recto appendiculatis, stylo glabro ca. $6 \mathrm{~mm}$. longo, ovario 5-loculari glabro vertice rotundato. Capsula cum calycis limbo $8 \mathrm{~mm}$. longa campanulata, seminibus praematuris $2 / 3 \mathrm{~mm}$. longis brunneis cuneatis apice uno latere patulerostratis. P. Ledermannii MANSF. affinis, a qua differt foliorum laminis minoribus 5-plinerviis, paniculae axibus teretibus, pędicellis longioribus, calycis limbo vix lobato.

No. 13468 Kanehira-Hatusima, Angi, Arfak Mts., on edge of mossy forests along the track to the Lake Gita from Momi, alt. about $1700 \mathrm{~m}$. above the sea, April 5, 1940. Flowers pink.

\section{Poikilogyne rubro-suffusa OHwI, sp. nov.}

Rami alato-4-quetri glabri 4-5 mm. diametientes, nodis crassiusculis. Folia opposita aequalia, (cum petiolo 20-40 mm. longo) 10-15 cm. longa 6-9 cm. lata subcoriacea, juventute parce furfuracea, demum glabrata, 
supra atroviridia, subtus pallidiora, nervis nervulisque elevatis rubescentia, 5-7-plinervia late elliptica vel cordato-orbicularia, apice obtusa vel obtusissima, basi cordata vel subcordata, margine densiuscule mucronato-serrulata, nervulis transversalibus manifestis, petiolis imprimis juventute supra furfuraceis. Panicula ampla terminalis, pedunculo excepto $15-20 \mathrm{~cm}$. longa et lata pyramidata laxe sed agglomerato-multiflora glabra, axibus subangulatis, pedicellis $2-4 \mathrm{~mm}$. longis parce furfurcaceis, medio saepe minutissime bibracteolatis. Flores 5-meri, calyce obconico vix furfuraceo $4 \mathrm{~mm}$. longo, limbo subdilatato vix $1 \mathrm{~mm}$. longo obsolete 5-lobo, lobis sub apice obsolete mueronulatis, petalis 5 earnosulis glabris oblique obovatis $8 \mathrm{~mm}$. longis subtruncatis, uno latere superne mucronatis. Stamina 10 aequalia flavida, filamentis $4 \mathrm{~mm}$. longis, antheris late lanceolatis obtusulis $3.5 \mathrm{~mm}$. longis, basi antice rotundatis inappendiculatis, postice tuberculo 1 ovato $0.5 \mathrm{~mm}$. Iongo obtuso unigibbosis, stylo $7 \mathrm{~mm}$. longo, ovario 5-loculari, vertice rotundato. Capsula $6 \mathrm{~mm}$. longa, semrinibus ignotis. Differt a P. Ledermannii Mansf., ramis tetrapteris, foliis latioribus, apice obtusis vel obtusissimis, vix angustatis, et a specie precedente, fere eodem necnon calycibus obconicis, pedicellis brevibus.

No. 13417 Kanehira-Hatusima, Angi, Arfak Mts., on edge of forests along the track to the Lake Angi from Momi, alt. about $1300 \mathrm{~m}$. above the sea, April 4, 1940. Flowers rose-coloured.

6. Poikilogyna arfakensis Baker fil. in GibBs, Contr. Fl. Phytog. Arfak Mts. (1917) 157; Mansf. in EngL. Bot. Jahrb. 60 (1926) 110.

Frutex $1 \mathrm{~m}$. altus (ex coll.), omnibus partibus pilis strictiusculis patentibus, saltem juventute rubescentibus apice atro-capitatis dense hirtus. Rami teretes vel obsolete angulati satis crassi. Folia late vel orbiculatoovata cum petiolo $3-4 \mathrm{~cm}$. longo $10-15 \mathrm{~cm}$. longa $6-8 \mathrm{~cm}$. lata herbacea, apice acutiuscula vel obtusula, basi cordata vel rotundata, 5-7-plinervia, nervis, nervulis transversalibus, nervulis irregularibusque subtus elevatis et saepe rubescentibus notata, margine obtuse dentata. Panicula terminalis inferne foliosa pyramidata subdense multiflora, $10 \mathrm{~cm}$. lata, ramis patentibus versus apicem floriferis, pedicellis $5-8 \mathrm{~mm}$. longis. Flores 6 -meri (rarius 5-meri), calyce anguste obconico 5-6 mm. longo dense hirto, limbo leviter obtuse-lobato, petalis $7-8 \mathrm{~mm}$. longis patentibus oblique ellipticis, apice rotundatis excentrice apiculatis. Stamina 12 aequalia, filamentis $4 \mathrm{~mm}$. longis, antheris late lanceolatis flavis apice obtusulis, appendice excepta $3 \mathrm{~mm}$. longis, basi antice rotundatis inappendiculatis, postice tuberculo 1 crasso $1 \mathrm{~mm}$. longo praeditis, ovario glabro vertice rotundato, stylo glabro $8 \mathrm{~mm}$. longo. Planta omnibus partibus pilis apice atro-capitatis obsitis valde insignis. 

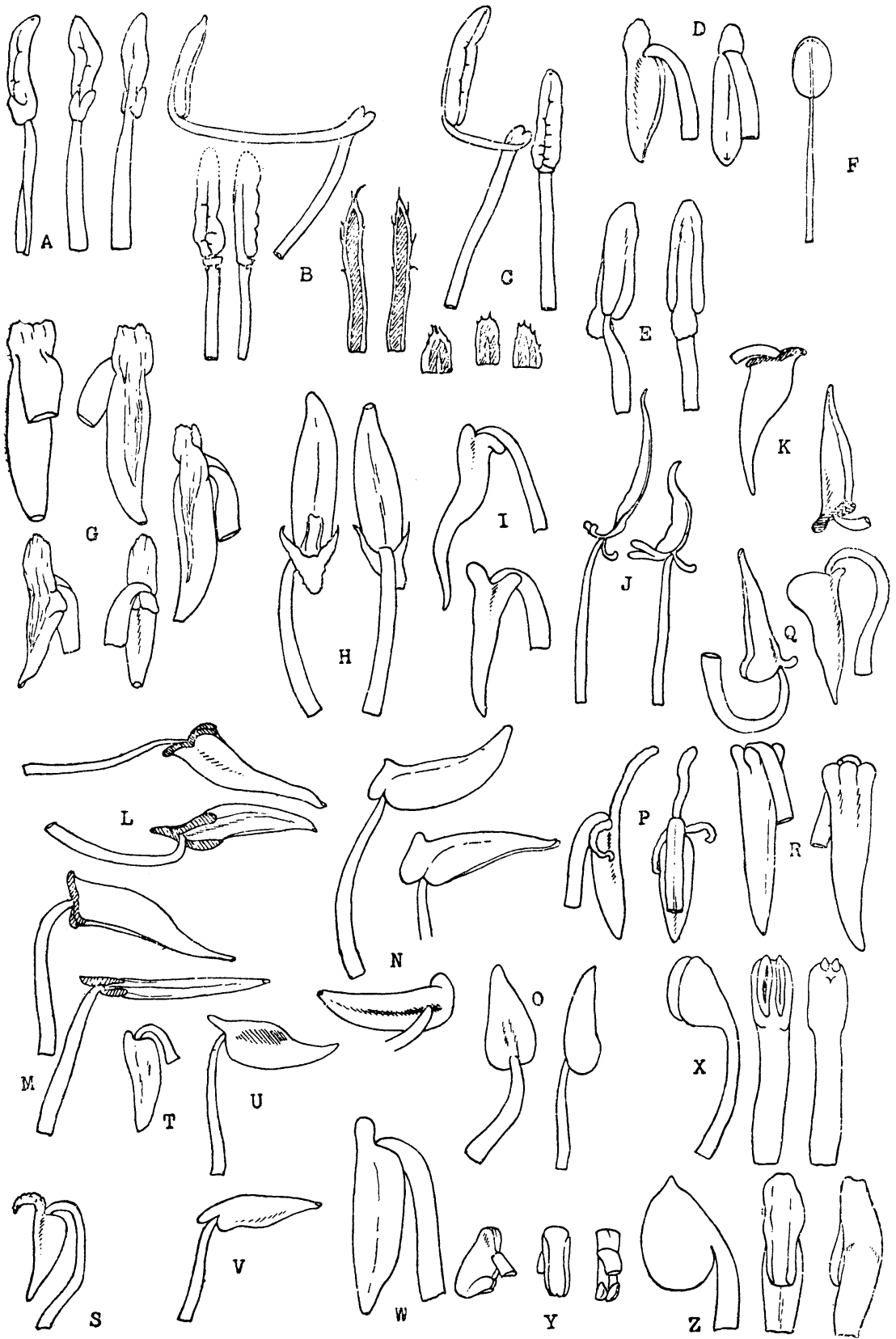

Stamina (et setae calycis in Melastomatibus) Melastomacearum.-A Otanthera novoguineensis. B Melastoma patulisetum. C Melastoma Roemeri. D Poililogyne rubrosuffusa. E Poililogyne arfakensis. F Sonerila papuana. G Phyllapophysis Schlechteri. $\mathrm{H}$ Dissochaeta angiensis. I Medinilla quintuplinervis. J Medinilla quadrifolia. $\mathrm{K}$ Medinilla crassiuscula. L Medinilla boemiensis. M Medinilla pachyphylla. N Medi- 
nilla trinervia. O Medinilla gibbifolia. P Medinilla tetraptera. Q Medinilla longifolia. R Medinilla annulifera. S Medinilla arfatensis. T Medinilla papulosa. U Medinilla dalmannensis. V Medinilla ferruginescens. W Astronidium subvaginatum. X Astronia papuana. Y Astronia Hatusimae. Z Astronia lanceata.

No. 14089 Kanehira-Hatusima, Angi, Arfak Mts., on the burnt open summit of Mt. Koebre, alt. $2200 \mathrm{~m}$. above the sea, April 9, 1940. Flowers rose coloured.

Distr. Endemic.

7. Sonerila papuana Cogn. in DC. Monogr. Phaner. 7 (1891) 510; Mansf. in Engl. Bot. Jahrb. 60 (1926) 112.

Antherae èllipticae $1.5 \mathrm{~mm}$. longae utrinque rotundatae, semina $0.5 \mathrm{~mm}$. longa oblonga pallide brumnea nitida teretia, apice parcissime subpapillosa, raphe laterali lineari concava, superne supra raphem grosse unituberculata.

No. 11889 Kanehira-Hatusima, Chaban, $30 \mathrm{~km}$. inward of Nabire, in rain-forests, alt. about $300 \mathrm{~m}$. above the sea, Febr. 29, 1940. Flowers white.

Distr. Endemic.

8. Phyllapophysis Schlechteri MANsF. in ENGL. Bot. Jahrb. 60 (1926) 114.

Petioli 15-20 mm. longi, pedunculi uniflori 5-7 cm. longi, apice bracteis 4-6 linearibus 6-8 mm. longis incurvis praediti, pedicellis $10-20 \mathrm{~mm}$. longis sursum incrassatis, petala late orbicularia sicco nigricantia $12 \mathrm{~mm}$. longa $15 \mathrm{~mm}$. lata carnosula, margine parce papulosa, staminum majorum antherae basi postice lamella quadrata apice erosula, minorum antherae basi antice utrinque lobulo ovato, postice lamella erosula oblonga appendiculatae, fructus baccatus subglobosus, apice truncatus.

No. 12749 Kanehira-Hatusima, Boemi, Nabire, in Agathis-forests, alt. about $400 \mathrm{~m}$. above the sea, March 11, 1940. Climbing, flowers white.

Distr. Endemic.

9. Dissochaeta angiensis KaneHiRA et Hatusima, sched.

Frutex scandens $5 \mathrm{~m}$. altus (ex coll.), omnibus partibus praeter foliorum laminam supra glabram pilis ferrugineis stellatis dense vestitus demum partim glabrescens. Rami elongati teretes vel subteretes. Folia oblongoovata chartacea, supra viridia, cum petiolo $12-18 \mathrm{~cm}$. longa 4-7 cm. lata, 5-plinervia, supra costa 1 tantum impressa notata, supra parce obsoleteque albo-punctulata, subtus nervis(5) nervulisque transversalibus (pluribus) elevatis notata, apice breviter caudato-obtusula, basi subcordata, petiolis 10-12 mm. longis. Panicula terminalis et e nodis superioribus axillaris, cum pedunculo basi globoso-incrassato $(2-4 \mathrm{~cm}$. longo) $8-12 \mathrm{~cm}$. longa pluri- 
flora nutans, pedicellis quam calyx subaequilongis. Flores 4-meri, calyce tubuloso-campanulato stellato-ferrugineo-tomentoso $5 \mathrm{~mm}$. longo, sub limbo satis contracto, limbo undulato-4-denticulato, petalis 4, oblongis glabris 8 mm. longis obtusis. Stamina 4 aequalia, filamentis flavidis $5 \mathrm{~mm}$. longis, apice in anthesi reflexis, antheris lanceolatis fusco-purpurascentibus apice pallidioribus obtusulis uniporosis $5 \mathrm{~mm}$. longis, a dorso plus minus compressis, inferne postice bistriatis, antice (vel utrinque latere) setis 2 subulato-compressis luteis obsolete denticulatis vix $1 \mathrm{~mm}$. longis acutis, basi postice lamella plana ovata obtusa lutea vix $1 \mathrm{~mm}$. longa praeditis, ovario ex toto fere adhaerente, vertice glabro planiusculo, stylo glabro 10-12 mm. longo, stigmate vix incrassato punctulato.

No. 13374 Kanehira-Hatusima, Angi, Arfak Mts., on edge of rainforests along the track to the Lak Gita from Momi, alt. about $1300 \mathrm{~m}$ above the sea, April 4, 1940.

10. Dissochaeta deusta OHwI, sp. nov.

Frutex scandens $4 \mathrm{~m}$. altus (ex coll.), omnibus partibus praeter foliorum laminam supra glabram pilis atro-fuscis stellatis praeditus. Rami elongati dense pilosi teretes vel subteretes, pilis partim decolorantibus. Folia ovata chartacea cum petiolo 9-11 cm. longa 4-5 cm. lata 5-plinervia, apice breviter acuminato-subulata, basi subcordata, supra costis 1 tantum leviter impressis notata, punctis minutis elevatis deniuscule obsita, subtus pilis praesertim in nervis nervulisque (elevatis) densius obsita, subtus lineolis elevatulis conspicue reticulata, petiolis $6-8 \mathrm{~mm}$. longis. Panicula terminalis sessilis efoliata $7 \mathrm{~cm}$. longa pyramidata pluriflora, axi angulato, pedicellis 6-8 mm. longis supra medium saepe uninodis, ovario apice stellatopiloso, rotundato-emargináto. Bacca oblongo-ovoidea teres $1 \mathrm{~cm}$. longa cernua, apice sensim contracta, calycis limbo aperto 7-8 $\mathrm{mm}$. lato brevissime 4-lobo vix mucronato intus etiam stellato-piloso coronata, seminibus $2 / 3 \mathrm{~mm}$. longis cuneatis angulatis, a latere subcompressis, truncatis, uno latere supra raphem lateralem patule rostratis glabris laevibus.

No. 11999 Kanehira-Hatusima, Dallmann, Nabire, in Agathis-forests, alt. about $400 \mathrm{~m}$. above the sea, March 1, 1940.

11. Medinilla quintuplinervis Cogn. in DC. Monogr. Phaner. 7 (1891) 574; Mansf. in Engl. Bot. Jahrb. 60 (1926) 119.

A descriptione originali paullo diversa : foliis $15-20 \mathrm{~cm}$. longis $6-7 \mathrm{~cm}$. latis apice breve acuminatis, basi breviter attenuato-acutis, petiolis 15-30 $\mathrm{mm}$. longis, floribus subfasciculatis vel subsolitariis.-Rami brunneo-cinerei, folia supra atro-, subtus subglauco- vel luteo-viridia, anguste oblonga, subtus opaca, pedicelli 4-5 mm. longi, flores 6-meri, petalis ante anthesin $7 \mathrm{~mm}$. 
longis ellipticis, staminibus 12, subinaequalibus, antheris 3 et $5 \mathrm{~mm}$. longis basi postice breviter oblique calcaratis, antice obtuse biauriculatis, ovarium glabrum, vertice convexo-rotundatum, stylo crasso glabro.

Sine num. Kanehira-Hatusima. Patema, Nabire, alt. about $300 \mathrm{~m}$. above the sea, 1940. Scandent in rain-forests.

Distr. Endemic.

12. Medinilla crassinervia Blume in Flora (1831) 510 et in Rumphia $1: 15$; Mansf. in ENGL. Bot. Jahrb. 60 (1926) 119.

Cymae 2-4-nae, 3-florae, pedunculis 10-20 mm. longis, pedicellis 8-15 $\mathrm{mm}$. longis saepe medio bibracteolatis, bracteolis deciduis, bacca $10-13 \mathrm{~mm}$. diametiens, apice calycis limbo truncato vix 5-mucronato circumdata, semina lutescentia laevia paullo compressa $1 \mathrm{~mm}$. longa dimidiato-obdeltoidea, raphe medio longitudinaliter convexa.

No., 11700 Kanehira-Hatusima, Papaya, Nabire, alt. about $150 \mathrm{~m}$. above the sea, Febr. 28, 1940. Scandent in rain-forests.

Distr. New Guinea, Ins. Banda, Ins. Ternate, Borneo, Malacca.

13. Medinilla quadrifolia BL. in Flora (1831) 509; Cogn. in DC. Monogr. Phanerog. 7 (1891) 574; Mansf. in EngL. Bot. Jahrb. 60 (1926) 119.

Folia coriacea $6-8 \mathrm{~cm}$. longa $2-3 \mathrm{~cm}$. lata, supra atroviridia, subtus ferruginescentia, petiolis $8-10 \mathrm{~mm}$. longis, flores 4-meri, pauci subcymosi vel simplices, pedicellis 10-20 mm. longis medio 1-2-nodosis, calyce $6 \mathrm{~mm}$. longo, limbo truncato, petalis $10 \mathrm{~mm}$. longis, staminibus subinaequalibus, filamentis $7-8 \mathrm{~mm}$. longis, antheris brevioribus lanceolatis acutis, (cum calcaribus 3 basillaribus $1.5 \mathrm{~mm}$. longis ascendentibus) $6 \mathrm{~mm}$. longis, connectivo basi non producto, antheris longioribus (cum calcaribus 3 basilaribus $1.5 \mathrm{~mm}$. longis arcuato-ascendentibus) $10 \mathrm{~mm}$. Iongis linearibus, connectivo basi levissime (1 mm. longo) producto, stylo $10 \mathrm{~mm}$. longo glabro, bacca urceolata 10-13 mm. longa 9-12 mm. lata, semina $1 \mathrm{~mm}$. longa dimidiato-obdeltoidea nitida laevia, a latere compressa, cellulis extimis minutis obsoletis nec elevatis nec impressis, taphe laterali elongata.

No. 12207 Kanehira-Hatusima, Dallmann, Nabire, alt. about $400 \mathrm{~m}$. above the sea, March 2, 1940. Scandent on edge of Agathis-forests, flowers pink, fruit rose-coloured.

Distr. New Guinea, Java, and Sumatra.

14. Medinilla rubrifructus $\mathrm{OHw}_{\mathrm{H}}$, sp. nov.

Frutex glaberrimus epiphyticus. Rami satis tenues rufo-brunnescentes teretes, nodis paullo incrassatis. Folia anguste oblonga vel anguste ovata 
opposita tenuiter chartacea brunneo-viridia triplinervia 7-10 cm. longa 2.5$4 \mathrm{~cm}$. lata, supra lucidula vix nervosa, subtus elevato-costata, subsessilia, apice in acumen obtusulum attenuata, basi obtusa vel subrotundata, nervis transversalibus vix visibilibus, petiolis $1 \mathrm{~mm}$. longis. Flores pauci-fasciculati axillares 4 -meri, pedicellis $2-3 \mathrm{~mm}$. longis tenuibus vix furfuraceis, basi minute subulato-bracteatis, calycis limbo humili obsolete 4-lobo, lobis incrassatis atratis sub apice brevissime mucronatis. Bacca sphaeroidea glabra teres 3-4 mm lata, vertice margine acuto late truncata (1.5 $\mathrm{mm}$ diametiens), calycis limbo aequialta. Semina $0.7 \mathrm{~mm}$ longa oblique oblonga, areolis elevatis minute reticulata, raphe laterali fusco-resinoso. Differt a M.Schumanniana Mansf., ramis vix verrucosis, foliis minoribus vix distincte petiolatis, pedicellis brevioribus.

No. 12662 Kanehira-Hatusima, Ayerjat, $40 \mathrm{~km}$. inward of Nabire, about $300 \mathrm{~m}$. above the sea, April 9, 1940. Epiphytic in fringing rainforests, about $80 \mathrm{~cm}$. tall, fruit red.

\section{Medinilla crassiuscula OHwi, sp. nov.}

Frutex ca. $30 \mathrm{~cm}$. altus epiphyticus glaber. Rami teretes vel obsolete tetragoni radicantes brunnei vel brunneo-cinerei parcissime lenticellati 2-2.5 mm. crassi. Folia opposita aequalia coriacea crassiuscula elliptica obscure viridia triplinervis, apice obtusa, basi obtusa vel acutiuscula, cum petiolo $10-12 \mathrm{~cm}$. longa 5-8 cm. lata, nervis subtus vix conspicuis, supra leviter elevatis, nervulis transversalibus supra tantum visibilibus, petiolis 3-5 mm. longis complanatis. Flores 4-meri pauci fasciculati laterales, pedicellis $2-4 \mathrm{~mm}$. longis vix furfuraceis, calycis tubo fere sphaeroideo, apice constricto et in limbum truncatum demum irregulariter erosulum dilatato, videtur rubescente, parce furfuraceo vel glabrescente, petalis 4 latissime deltoideo-orbicularibus crassiusculis in alabastro $2 \mathrm{~mm}$. longis obtusis. Stamina 8 aequalia, antheris flavidis, apice acutis, basi rubra truncatis, stylo glabro, ovario 4-loculari. Bacea immatura 3-4 mm. longa et lata, calycis tubum non superans, seminibus ovoideis $0.8 \mathrm{~mm}$. longis leviter obliquis, areolis elevatis minutis reticulatis, raphe laterali. Differt a $M$. tenuipedicellata BAK. f., foliis majoribus, petiolis vix conspicuis, et a $M$. novoguineensi, foliis triplinerviis.

No. 12353 Kanehira-Hatusima, Patema, Nabire, in rain-forests, about $300 \mathrm{~m}$. above the sea, March 5, 1940. Epiphytic.

16. Medinilla rubrifolia Mansf. in ENGL. Bot. Jahrb. 60 (1926) 122.

Specimina foliorum forma satis variabilia, ramis satis crassis.-Rami lenticellis densiusculis subverrucosi teretes, folia sessilia vel fere sessilia percoriacea atroviridia, subtus rubro- vel ferrugineo-suffusa, apice acuta vel obtusa vel angustato-obtusula $15-30 \mathrm{~cm}$. longa 6-10 cm. lata, cum nervo 
marginali breviore tenuioreque 5-plinervia, nervis subtus elevatis, nervulis transversalibus supra tantum obsolete visibilibus notata, subtus opaca, supra vix nitida, basi cuneata usque rotundata, flores fasciculati 4-meri, fructibus praematuris $4 \mathrm{~mm}$. latis et longis, vertice rotundato calycis tubum leviter superantibus rubris.

No. 12251, 12274 Kanehira-Hatusima, Dallmann, $45 \mathrm{~km}$. inward of Nabire, in Agathis-forests, about 400-500 m. alt. above the sea, March 3, 1940. Epiphytic, $50-80 \mathrm{~cm}$. tall, fruits red.

Distr. Endemic.

17. Medinilla fasciculifiora Онwi, sp. nov.

Frutex $1 \mathrm{~m}$. altus glaberrimus. Rami satis crassi teretes lenticellati cinereo-brunnei. Folia opposita coriacea sessilia vel subsessilia ovata, nervo marginali tenuiore excepto subtriplinervia, opaca supra atroviridia, subtus sufescenti-viridia, costis supra levissime, subtus prominente elevata, nervulis transversalibus supra tantum obsolete visibilibus notata apice obtusa, basi rotundata, ca. $12 \mathrm{~cm}$. (cum petiolo) longa $6-7 \cdot \mathrm{cm}$. lata, late ovata vel ovata. Flores axillares fasciculati vix bracteati, 4-meri, pedicellis subtenuibus rubris glabris $8-10 \mathrm{~mm}$. longis, calyce urceolato glabro atro-rubro, in fructu juvenili 4-5 mm. longo 3-4 mm. lato, basi latissime obconico, limbo $1 \mathrm{~mm}$. longo levissime undulato-4-lobo, lobis vix apiculatis. Bacca apice rotundata calycis tubum vix superans, seminibus oblique anguste obovatis $0.8 \mathrm{~mm}$. longis laevibus nitidis, raphe laterali, medio leviter elevato-unistriato. $M$. rubrifoliae affinis, a qua differt foliis minoribus basi rotundatis, calyce laevi, fructu apice calycis limbum vix superante. A $M$. crassiuscula, ramis densius lenticellatis, foliis rotundatis, costis subtus prominentibus, pedicellis longioribus diversa.

No. 13649 Kanehira-Hatusima, Angi, Arfak Mts., in forests on the western ridge running up to the Lake Gita, alt. about $200 \mathrm{~m}$. above the sea, April 6, 1940. Epiphytic.

18. Medinilla boemiensis Kanehira et Hatusima, sched.

Frutex terrestris $1 \mathrm{~m}$. altus glaberrimus, videtur basi repens. Rami elongati teretes densiuscule lenticellati subtenues, cinereo-brunnei, inferne sparse radicans, nodis levissime incrassatis. Folia opposita late lanceolata subcoriacea, supra atroviridia, subtus flavovirentia, cum petiolo brevissimo (1-2 mm. longo) 15-17 cm. longa 4-5 cm. lata, praeter nervos marginales tenuissimos triplinervia, utrinque attenuata, apice tamen obtusiuscula, basi acuta sessilia vel subsessilia, nervulis transversalibus vix visibilibus, costis subtus tantum elevatis. Cymae axillares 10-15-florae, pedunculis 5-10 mm. longis tenuibus semel bisve ternato-ramosis, bracteolis subulatis minimis, 
pedicellis gracilibus $15-20 \mathrm{~mm}$. longis. Flores 4-meri, albi (ex coll.), calyce brevicampanulato $3 \mathrm{~mm}$. longo, limbo truncato-eroso sub apice obsolete 4-mucronulato, petalis 4 ellipticis obtusis $7 \mathrm{~mm}$. longis. Stamina 8 aequalia flavescentia, filamentis $4-5 \mathrm{~mm}$. longis, antheris lanceolatis attenuato-acutis $4 \mathrm{~mm}$. longis uniporosis, a latere plus minus compressis, basi rubro-brunnescente crassa oblique truncatis, postice basi acuto-productis, antice inappendiculatis, stylo glabro, ovario glabro vertice rotundato. Bacea rubra $5 \mathrm{~mm}$. longa $4 \mathrm{~mm}$. lata, seminibus oblongo-obovatis $0.7 \mathrm{~mm}$. longis, cellulis minutis vix elevatis punctulatis, raphe laterali medio leviter elevato-unistriata. A M. Ledermannii MANsF., foliis basi angustatis, floribus subcymosis, pedicellis longioribus differt.

No. 12728 Kanehira-Hatusima, Boemi, $40 \mathrm{~km}$. inward of Nabire, Geelvink Bay, in Agathis-forests on a ridge, alt. $300 \mathrm{~m}$. above the sea, March 10, 1940.

19. Medinilla pachyphylla Kanehira et Hatusima, sched.

Frutex glaberrimus epiphyticus ca. $1 \mathrm{~m}$. altus. Rami satis crassi teretes valde lenticellato-verrucosi et longitudinaliter demum sulcati, vetusti serius radiculosis. Folia sessilia opposita oblongo-obovata $30 \mathrm{~cm}$. longa $15 \mathrm{~cm}$. lata coriacea crassa opaca 5-plinervia, apice abrupte contracta, obtusa (?), nervis subtus elevatis, nervulis tranversalibus magis obliquiis vix visibilibus notata, basi cuneata. Flores 4-meri, calyce campanulato juventute furfuraceo $3 \mathrm{~mm}$. longo rubro, sub limbo satis constricto, limbo 1-1.2 $\mathrm{mm}$. longo truncato demum irregulariter $4-5$-lobo, petalis 4 obovatis $7-8 \mathrm{~mm}$. longis albis (ex coll.). Stamina 8 aequalia flavescentia, filamentis quam antherae aequilongis, artheris lanceolatis $4 \mathrm{~mm}$. longis apice attenuatis, basi rubrobrumnea oblique truncatis, a latere modice compressis, ovario glabro, vertice late rotundato-subtruncato, stylo glabro $3-4 \mathrm{~mm}$. longo. Bacca $4 \mathrm{~mm}$. lata, cum calycis limbo basi contracto $5 \mathrm{~mm}$. longa, vertice calycis tubum non excedens, seminibus obovatis $0.6 \mathrm{~mm}$. longis, cellulis elevatis punctulatis, raphe laterali oblonga medio longitudinaliter convexa. Ex descriptione affinis M.rubrifoliae MANsF., sed ramis dense radiculosis, foliis latioribus, calyce verrucis destituto, antheris, filamentis, petalisque longioribus.

No. 12171 Kanehira-Hatusima, Dallmann, $45 \mathrm{~km}$. inward of Nabire, in Agathis-forests, alt. about $500 \mathrm{~m}$. above the sea, March 2, 1940.

20. Medinilla papulosa OHwi, sp. nov.

Frutex epiphyticus. Rami teretes ferruginei sparsim verrucosi, inferne ascendentes et radicantes, juventute sparsim furfuracei. Folia oblonga opposita aequalia 5-plinervia sessilia glaucescentia, tenuiter chartacea, utrinque opaca, supra dense sed minutissime papulosa, subtus videtur pur- 
purascentia, utrinque sparsim furfuracea, glabrescentia, 10-12 cm. longa 4-5 cm. longa 4-5 cm. lata, apcie acutiuscula, basi acuta vel cuneata, costis subtus tantum elevatis verruculosis. Fasciculi florum oliganthi axillares, bracteis minimis subulatis, pedicellis $3-4 \mathrm{~mm}$. longis parce furfuraceis tenuibus. Flores albi (ex coll.) 4-meri, calyce tubuloso-campanulato $3 \mathrm{~mm}$. longo, limbo truncato vix mucronato, petalis 4 ovatis obtusis $3-4 \mathrm{~mm}$. longis glabris. Stamina 8 aequalia, antheris fere $2 \mathrm{~mm}$. longis, bas postice brevi-unicalcaratis, antice rotundato inappendiculatis. Ex clave Mansfeldiana (in ENGL. Bot. Jahrb. 60 : 115-118, 1926), vicina M. rubrifoliae, a qua diversissima foliis duplo minoribus glaucescentibus, pedicellis brevibus, ut calyx non verruculosis.

No. 12369 Kanehira-Hatusima, Patema, Nabire, in fringing rainforests, alt. about $500 \mathrm{~m}$. above the sea, 1940. Epiphytic, flowers white, leaves purpurascent beneath.

21. Medinilla dallmannensis Kanehira et Hatusima, sched.

Frutex glaber, ramosus. Rami teretes $1.5-2 \mathrm{~mm}$. diametientes fusci demum cito cinerascentes, nodis ad basin petiolorum pulvino dilatato muniti, supra nodos dense setosi (setis ca. $1 \mathrm{~cm}$. longis flexuosis). Folia opposita valde disparia vel quasi alternantia, minora late elliptica sessilia usque ad $3 \mathrm{~cm}$. longa, majora oblonga pallide viridia praeter nervos marginales tenuiores 5-plinervia, jumiora subtus ad costas tantum parce furfuracea cito glabrata subcoriacea, basi obtusa vel acuta, apice acuta vel breviter abrupteque acuminata, cum petiolo $10-15 \mathrm{~cm}$. longa $3.5-4.5 \mathrm{~cm}$. lata, costa supra vix impressis, subtus prominulis, nervulis vix visibilibus, petiolis 3-6 mm. longis. Cymae umbellatae 3-6-florae terminales solitariae, pedunculis communis ca. $15 \mathrm{~mm}$. longis, pedicellis anthesi $3-4 \mathrm{~mm}$., fructu $8 \mathrm{~mm}$. longis primo furfuraceis, bracteis subulato-ovatis $2-4 \mathrm{~mm}$. longis persistentibus. Flores 6-meri, calyce campanulato $3-4 \mathrm{~mm}$. longo fere glabro, limbo truncato breviter 6 -setuloso, petalis 6 late obovatis obtusis albis (ex coll.) $8 \mathrm{~mm}$. longis. Stamina 12, aequalia, filamentis $4 \mathrm{~mm}$. longis, antheris oblongo-ovatis $4 \mathrm{~mm}$. longis flavidis a latere compressis, apice acutis 1porosis, basi antice inappendiculatis, postice breviter calcaratis, ovario fere ex toto adhaerente 6-loculari, vertice planiusculo glabro exannulato, stylo glabro $6 \mathrm{~mm}$. longo. Bacea globosa $5-6 \mathrm{~mm}$. longa et lata, seminibus oblique obovatis laevibus nitidis $0.8 \mathrm{~mm}$. longis, raphe laterali medio linea 1 leviter elevata notata. Affinis $M$. Lauterbachianae Mansf., sed ramulis nodisque teretibus, foliis minoribus brevius petiolatis, floribus 6 -meris, calyce 6 setuloso.

No. 12107 Kanehira-Hatusima, Dallmann, Nabire, alt. about $400 \mathrm{~m}$. above the sea, in Agathis-forests, March 1, 1940. Epiphytic, about $1 \mathrm{~m}$. 
tall, flowers white, inflorescences rose-coloured.

22. Medinilla trinervia Cogn. in DC. Monogr. Phanerog. 7 (1891) 596 ; Mansf. in ENGL. Bot. Jahrb. 60 (1926) 125.

Frutex terrestris (?), $2 \mathrm{~m}$. altus glaberrimus. Rami cinerei satis graciles ramosi tetraquetri, nodis interdum papillosis. Folia sessilia ovata vel oblongo-ovata, apice longe acuta vel attenuata, basi subcordata vel rotundata opposita aequalia vel paullo disparia $3-8 \mathrm{~cm}$. longa $2-3 \mathrm{~cm}$. lata chartacea triplinervia, nervis tenuibus. Cymae terminales pauciflori, pedunculis $5-7 \mathrm{~mm}$. longis semel ternatoramosis, pedicellis 5-6 mm. longis tetragonis. Flores 5-meri, calyce campanulato $3 \mathrm{~mm}$. longo sub limbo contracto, limbo truncato 5-mucronulato, petalis.... Stamina 10, aequalia, filamentis $2.5 \mathrm{~mm}$. longis, antheris $2 \mathrm{~mm}$. longis flavidis, late lanceolatis, basi antice inappendiculatis rotundatis, postice supra basin obsolete 1-gibbosis, ovario fere ex toto adhaerente glabro, stylo glabro $5 \mathrm{~mm}$. longo. Fructus ignotus. Differt a descriptione originali floribus 5-meris.

No. 13958 Kanehira-Hatusima, Angi, Arfak Mts., in mossy forests along the Iray River, Lake Giji, alt. about $1900 \mathrm{~m}$. above the sea, April 8, 1940.

Distr. New Guinea.

23. Medinilla gibbifolia OHwI, sp. nov.

Pogonanthera gibbifolia OHw , sched.

Frutex glaber. Rami teretes crassiusculi sparsim lenticellati brumnei, cum petiolisque obsolete granuloso-rugulosi. Folia opposita aequalia oblonga vel obovato-oblonga subcoriacea, supra atro-, subtus ferrugineoviridia, praeter nervos marginales tenuibus triplinervia, cum petiolo 1-2 cm. longo $15-20 \mathrm{~cm}$. longa 5-8 cm. lata, apice obtusula vel abrupte acuta, subtus dense papillosa, costis utrinque prominulis, nervulis transversalibus supra tantum prominulis notata, basi obtusa et utrinque latere callo rotundato $2 \mathrm{~mm}$. lato et longo gibbosa. Panicula terminalis pedunculata deltoidea multiflora $6 \mathrm{~cm}$. longa et lata minutissime bracteata, ramis oppositis patentibus, pedicellis ca. $2 \mathrm{~mm}$. longis luteo-furfuraceis. Flores 4-meri, calyce $2-3 \mathrm{~mm}$. longo urceolato luteo-furfuraceo, limbo obtuse 5-dentato, petalis 4 carnosulis erectis ovatis obtusulis $3 \mathrm{~mm}$. longis extus luteo-furfuraceis. Stamina 8 aequalia, antheris oblongo-ovatis pallidis inappendiculatis $1.5 \mathrm{~mm}$. longis obtusulis, basi rocundatis, filamentis $1.5-2 \mathrm{~mm}$. longis, ovario 4-loculari fere ex toto adnato, vertice annulo intus luteo-pilosulo coronato, stylo $3 \mathrm{~mm}$. longo inferne parce furfuraceo. Bacca immatura globosa calycis limbo coronata $4 \mathrm{~mm}$. lata. Foliis basi utrinque gibbosis inter species valde insignis. Habitu fere Pogonantherae pulverulentae, a 
qua differt foliis subtus vix pallidis, staminibus glaberrimis, antheris basi inappendiculatis.

No. 12236 Kanehira-Hatusima, Dallmann, $45 \mathrm{~km}$. inward of Nabire, alt. about $400 \mathrm{~m}$. above the sea, in secondary forests, March 3, 1940. Terrestrial, about $2 \mathrm{~m}$. high, flowers orange-coloured.

\section{Medinilla tetraptera $\mathrm{OHWI}_{\mathrm{H}}$ sp. nov.}

Frutex epiphyticus glaber. Rami crassi tetraquetri, undulato-alati, supra nodos setis $2 \mathrm{~cm}$. longis densissime ornati. Folia opposita subaequalia sessilia obovato-oblonga $25-30 \mathrm{~cm}$. longa $10-15 \mathrm{~cm}$. lata, apice abrupte acuminata vel acuta, basi attenuata et anguste rotundata vel subcordata, supra viridia, subtus luteo-viridia subcoriacea, nervis utrinque 5-7 oppositis subtus tantum elevatis. Panicula terminalis multiflora, cum pedunculo brevi $7 \mathrm{~cm}$. longa $5 \mathrm{~cm}$. lata videtur cernua paŕce furfuracea 4 -angulata, ramis verticillatis, bracteis subulatis reflexis minutis, pedicellis $2-4 \mathrm{~mm}$. longis. Flores 5-meri, calyce aperte campanulato $3 \mathrm{~mm}$. longo truncato verruculoso glabro, limbo truncato-undulato, basi obsolete contracto, petalis 5 albis (ex coll.) late ovatis, paullo ante anthesin $4 \mathrm{~mm}$. longis, apice rotundatis, medio carnosulis. Stamina 10 aequalia, filamentis $3 \mathrm{~mm}$. longis, antheris calcaribus exceptis $3 \mathrm{~mm}$. longis flavis late lanceolatis, basi postice calcari rectiusculo filiformi paullo flexuoso $2 \mathrm{~mm}$. longo praeditis, antice calcaribus (2) $1.5 \mathrm{~mm}$. longis incurvis tenuibus praeditis, ovario glabro, apice conico, stylo glabro terminato 5-loculari. A M. Teysmanni MIQ. cui proxime affinis, differt autem foliis plerumque obovato-oblongis, supra medium latissimis, panicula minori, floribus minoribus, petalis albis minoribus, staminum antheris basi longius calcaratis.

No. 12261 Kanehira-Hatusima, Dallmann, Nabire, in Agathis-forests, alt. about $500 \mathrm{~m}$. above the sea, March 3, 1940. Epiphytic, flowers white, calyx red.

25. Medinilla longifolia Cogn. in DC. Monogr. Phaner. 7 (1891) 597 ; Mansf. in ENGL. Bot. Jahrb. 60 (1929) 128 et in Nova Guinea, 14 (1927) 206.

No. 12364 Kanehira-Hatusima, Patema, Nabire, in rain-forests, alt. about $300 \mathrm{~m}$. above the sca, March j, 1910. Infructescence red, seeds smooth. No. 11861 Kaneiria-IIATUidid, Chaban, Nabire, in rain-forests, alt. about $300 \mathrm{~m}$. above the sea, Febr. 28, 1940. About $1 \mathrm{~m}$. tall, flowers white, axis of the inflorescence red.

Distr. Endemic.

26. Medinilla annulifera OHwi, sp. nov. 
Specimen mancum, foliis glabris sessilibus sub-7-plinerviis, basi cuneatis, panicula pluriflora parce furfuracea, ramis verticillatis subebracteatis, pedicellis $2-3 \mathrm{~mm}$. longis, medio saepe nodosis, floribus 4-meris albis (ex coll.), calyce campanulato 4-5 mm. longo glabro, basi obconico, limbo truncato demum irregulariter $4-5$-lobo, petalis $5-6 \mathrm{~mm}$. longis ellipticis glabris, apice obtusis, medio carnosulis, staminibus 8 aequalibus, filamentis $3 \mathrm{~mm}$. longis, antheris flavidis lanceolatis $5 \mathrm{~mm}$. longis acutis uniporosis, basi postice tuberculatis, tuberculis obtusis brevibus, antice bitnberculatis, tuberculis brevibus vix conspicuis, ovario calyce ad medium usque adhaerente, et septis usque ad apicem connexo glabro, vertice annulo glabro coronato, stylo $5 \mathrm{~mm}$. longo glabro.

Sine numero Kanehira-Hatusima, Angi, Arfak Mts., in low forests on the ridge running up to the Lake Gita, alt. about $2000 \mathrm{~m}$. above the sea, April 5, 1940. Flowers white.

Distr. Endemic.

27. Medinilla arfakensis BAK. f. in GrBbs, Contrib. Phytog \& Fl. Arfak Mts. (1917) 158.

A descriptione originali paullulo diversa, ramis manifeste 4 -alatis, foliis paullo brevioribus, antherae calcaribus pl. m. recurvis. Frutex. Rami acute tetraquetri, angulis ala integra cincti cinerei, juventute fusci et brunneofurfuracei cito glabrati, nodis dense setosis (setis $1 \mathrm{~cm}$. longis). Folia opposita aequalia vel subaequalia elliptica vel oblonga 7 -nervia chartacea opaca viridia, subtus pallidiora, primo furfuracea demum glabrata sessilia 10-15 cm. longa 5-6 cm. lata, apice abrupte acuminata vel acuta, basi sessilia attenuato-obtusa vel attenuato-rotundata, nervis subtus elevatis, nervulis transversalibus supra tantum levissime visibilibus. Panicula terminalis pyramidata $5-10 \mathrm{~cm}$. longa multiflora, usque ad $8 \mathrm{~cm}$. lata furfuracea, bracteolis minimis, ramis verticillatis, pedicellis $2-4 \mathrm{~mm}$. longis. Flores 5meri, calyce aperte campanulato $3 \mathrm{~mm}$. longo parcissime furfuraceo, limbo truncato, petalis 5 ellipticis obtusis glabris $7 \mathrm{~mm}$. longis. Staminibus 10 aequalibus, filamentis $5 \mathrm{~mm}$. longis, antheris late lanceolatis $3 \mathrm{~mm}$ longis, basi postice longiuscule curvato-calcaratis (calcare obsolete verruculoso), antice rotundato inappendiculatis, ovario glabro vertice convexo, stylo glabro $4 \mathrm{~mm}$. longo.

No. 14073 Kanehira-Hatusima, Angi, Arfak Mts., in forests by the Lake Gita, alt. about $1900 \mathrm{~m}$. above the sea, April 9, 1940. Epiphytic, flowers white, inflorescences rose-coloured.

Distrib. Endemic.

28. Medinilla Kanehirae OHwi, sp. nov. 
Frutex epiphyticus glaberrimus praeter ramorum nodos papillis brunneis $1 \mathrm{~mm}$. longis cinctos. Rami satis crassi alato-tetraquetri cinerei laeves. Folia lanceolata vel late lanceolata subcoriacea triplinervia flavovirentia, cum petiolo $2-2.5 \mathrm{~cm}$. longo $25-30 \mathrm{~cm}$. longa $6-7 \mathrm{~cm}$. lata, a medio sursum sensim angustata acuminata, basi rotundata, nervis utrinque elevatulis, nervulis non conspicuis. Panicula axillaris ad nodos ramorum vetustorum, angusta, $30 \mathrm{~cm}$. longa (cum pedunculo $8-10 \mathrm{~cm}$. longo) $6 \mathrm{~cm}$. lata glabra, ramis verticillatis tetragonis, cymose $3-6$-floris, ca. $3 \mathrm{~cm}$. longis, pedicellis 5-8 mm. longis, bracteis deciduis. Flores 4-meri. Bacea globosa 4-5 mm. longa et lata, apice calycis limbo depresso integro $1.5 \mathrm{~mm}$. lato cincta, seminibus teretiusculis oblongo-obovatis $1 \mathrm{~mm}$. longis laevibus nitidis, raphe laterali oblonga convexa. Habitu M. longifoliae Cogn. similis, a qua diversa nodis ramorum non longe setosis, foliis petiolatis basi magis rotundatis, paniculis ex ramorum vetustorum nodis gerentibus.

No. 12140 Kanehira-Hatusima, Dallmann, Nabire, alt. about $400 \mathrm{~m}$. above the sea, April 2, 1940. Epiphytic in Agathis-forests, about $1 \mathrm{~m}$. tall, infructescences red.

29. Medinilla ferruginescens $\mathrm{OHw}_{\mathrm{w}} \mathrm{sp}$. nov.

?Pogonanthera hexamera Bak. f. in GibBs, l. c. (1917) 158, non Medinilla hexamera BAK. f.

Frutex 1-1.5 m. altus (ex coll.), omnibus partibus, praeter laminam foliorum supra costis tantum pubescentem, pilis ferrugineis pinnatis dense furfuraceo-pubescentibus. Rami tenuiusculi teretes ramosi elongati. Folia opposita aequalia cum petiolo $10-15 \mathrm{~mm}$. longo $10-15 \mathrm{~cm}$. longa $3-6 \mathrm{~cm}$. lata oblonga vel anguste oblonga vel oblongo-obovata triplinervia, utrinque acuta vel basi breviter cuneato-attenuata, supra sub lente impresso-punctulata, nervis subtus tantum elevatis, nervulis transversalibus vix visibilibus. Panicula terminalis laxe pluriflora, cum pedunculo brevi $10-15 \mathrm{~cm}$. longa 7-10 cm. lata pyramidata, bracteis homomorphis persistentibus late lanceolatis $7-8 \mathrm{~mm}$. longis acutiusculis, pedicellis $4-5 \mathrm{~mm}$. longis. Flores 6 -meri, calyce brevi-campanulato $3 \mathrm{~mm}$. longo primo dense pubescente, limbo humile sed acutiuscule 6 -denticulato, petalis anguste oblongis reflexis glabris obtusis ca. $5 \mathrm{~mm}$. longis. Staminum antheris late lanceolatis acutis $2.5 \mathrm{~mm}$. longis, basi antice rotundatis inappendiculatis, postice breviter unituberculosis, filamentis $2.5 \mathrm{~mm}$. longis, ovario parce pubescente. Bacca globosa $5 \mathrm{~mm}$. longa et lata, calycis limbo $4 \mathrm{~mm}$. diametiente brevi coronata, seminibus permultis $0.8 \mathrm{~mm}$. longis obovato-oblongis nitidis, cellulis extimis vix elevatis non impressis, raphe lateraliter excurrente oblonga. Ex descriptione affinis videtur M. rubiginosae CogN., a qua diversa pilis non stellatis, inflorescentia terminali, petalis obtusis brevioribus. 
No. 13926 (Type), 13767, 13609 Kanehira-Hatusima, Angi, Arfak Mts., in forests near Iray by the Lake Giji, alt. about $1900 \mathrm{~m}$. above the sea, April 5-8, 1940. A shrub $1.5 \mathrm{~m}$. tall.

Distr. Endemic.

\section{Astronidium subvaginatum $\mathrm{OH}$ но, sp. nov.}

Creochiton subvaginata OHwI, schedul.

Arbuscula $10 \mathrm{~m}$. alta (ex coll.) glabra. Rami teretes vel obsoletissime tetragoni crassiusculi, fusci, demum cinereo-brunnei laeves, infra foliorum insertionem humile bicallosi, supra insertionem pilis simplicibus fuscis 2-3 mm. longis adpressis cincti. Folia oblonga opposita aequalia $15-20 \mathrm{~cm}$ longa (cum petiolo $3-5 \mathrm{~cm}$. longo), 5-8 cm. lata subcoriacea, supra atroviridia, vix opaca, subtus pallidiora et praesertim in nervis parce furfuracea, praeter nervos marginales tenuiculos triplinervia, apice acuta, basi acuta vel subacuta, costis nervisque transversalibus '(rubescentibus) pluribus supra vix impressis, subtus elevatis, nervulis anastomosantibus minutis subtus conspicuis notata, petiolis basi appendiculo ovato $5-8 \mathrm{~mm}$. longo adnato inconspicuo subvaginiformi cinctis. Panicula terminalis pluriflora subcorymbosa, cum pedunculo 4-5 cm. longo 7-10 $\mathrm{cm}$. longa 5-6 cm. lata sparse furfuracea, ramis oppositis ca. $3 \mathrm{~cm}$. longis, pedicellis $5 \mathrm{~mm}$. longis, flores 5-meri, calyce turbinato $8-10 \mathrm{~mm}$. longo 6-7 mm. lato, limbo truncato demum irregulariter lobulato, petalis 5 orbiculari-obovatis carnosulis obtusis 5-6 mm. longis. Stamina 10 aequalia, antheris $4.5 \mathrm{~mm}$. longis lanceolatis, basi postice breviter calcaratis, antice rotundatis inappendiculatis, filamentis $4 \mathrm{~mm}$. longis, ovario glabro vertice plano, stylo glabro, stigmate non incrassato, ovario 5-loculari, placenta basilari erecta cuneata, ovulis permultis.

No. 14105 Kanehira-Hatusima, Angi, Arfak Mts., in forests by the Lake Gita, alt. about $1900 \mathrm{~m}$. above the sea, April 10, 1940. A small tree, flowers white.

31. Astronia papuana Cogn. in DC. Monogr. Phanerog. 7 (1891) 1095.

Frutex. Rami teretes vel subteretes cinerascentes parce furfuracei vix lenticellati crassiusculi dense foliosi. Folia late oblanceolata vel anguste oblonga pallide viridia opaca subcoriacea, praeter nervos marginales tenuissimos triplinervia $20-25 \mathrm{~cm}$. longa $4-6 \mathrm{~cm}$. lata, utrinque acuta vel basi obtusula, supra glabra, subtus praecipue ad nervos furfuracea, costis subtus tantum elevatis, nervis transversalibus multis neryulisque subtus tantum visibilibus notata, petiolis $2-3 \mathrm{~cm}$. longis. Panicula terminalis corymbosa sessilis $8 \mathrm{~cm}$. longa et lata apice planiuscula multiflora dense brunneo-furfuracea, pedicellis $2-5 \mathrm{~mm}$. longis medio saepe subulato-bracteolatis, brac- 
teolis 2-3 mm. longis. Flores 5-meri, calyce obconico 4-5 $\mathrm{mm}$. longo dense furfuraceo, limbo 5-lobo intus parce furfuraceo, lobis deltoideis acutis 1.5 $\mathrm{mm}$. longis, petalis 5 late ellipticis carnosulis. Stamina 10 aequalia, filamentis $2 \mathrm{~mm}$. longis latiusculis, antheris a latere subcompressis $1 \mathrm{~mm}$. longis obovatis inappendiculatis, ovario glabro ex toto adhaerente vertice truncato vel subconcavo, stylo $3-4 \mathrm{~mm}$. longo, stigmate capitato.

No. 13283 Kanemra.Hatusima, Momi, 60 miles south of Manokwari, alt. about $20 \mathrm{~m}$. above the sea, in rain-forests, March 30, 1940. Plant $3 \mathrm{~m}$. tall, fiowers orange-coloured.

Distr. Endemic.

32. Astronia Hatusimae OHwi, sp. nov.

Arbuscula. Rami juniores subtetragoni, demum teretiuseuli cinereobrunnei sparse lepidoti, crassiusculi dense foliati. Folia opposita late lanceolata vel anguste oblonga chartacea opaca utrinque breve attenuata, apice acuminata, basi petiolata, supra viridia, glabra, subtus dense lepidota, ferruginea, triplinervia, nervis supra non impressis, subtus elevatis, nervulis transversalibus pluribus subtus tantum manifestis notata, petiolis subtenuibus $2-3 \mathrm{~cm}$. longis. Panicula terminalis corymbiformis multiflora $12 \mathrm{~cm}$. lata, cum pedunculo $1 \mathrm{~cm}$. longo $7 \mathrm{~cm}$. longa sparsim lepidotis, pedicellis $3-5 \mathrm{~mm}$. longis dense lepidotis ferrugineis. Flores 5 -meri, calyce $3 \mathrm{~mm}$. longo dense ferrugineo-lepidoto, basi rotundato, sub limbo leviter constricto, limbo כ-dentato, dentibus remotiusculis fere $1 \mathrm{~mm}$. longis acutis, petalis 5 . Stamina 10, filamentis brevibus, antheris obovatis a latere plus minus compressis flavidis basi obtuse et indistincte rotundatis, ovario truncato ex toto adhaerente biloculari, placentis 2 depressis, stigmate capitato. $\mathrm{Ab} A$. Beccariana CogN, differt, ramis vix acutiuscule tetragonis, foliis majoribus chartaceis, utrinque attenuatis, calyce dense lepidoto, dentibus acutis, ovario certe biloculari.

No. 12172 Kanehira-Hatusima, Dallmann, Nabire, in Agathis-forests, alt. about $500 \mathrm{~m}$. above the sea, March 2, 1940. Plant $5 \mathrm{~m}$. tall.

33. Astronia lanceata OHwı, sp. nov.

Arbuscula $8 \mathrm{~m}$. alta (ex coll.). Rami acute tetrangulati satis ramosi ferrugineofusci dense lepidoto-furfuracei. Folia lanceolata coriacea triplinervia cum petiolo $8-15 \mathrm{~mm}$. longo demum glabrato $8-12 \mathrm{~cm}$. longa $15-20$ $\mathrm{mm}$. lata apice breve acuminata, basi acuta petiolata, supra glabra, subtus dense ferrugineo-lepidota, nervis supra leviter impressis, subtus elevatis, nervulis transversalibus tenuibus subtus tantum visibilibus notata. Panicula terminalis $4-6 \mathrm{~cm}$. longa et lata pluriflora dense ferrugineo-lepidoto-furfuracea. Flores 4-meri, calyce urceolata dense lepidoto $3 \mathrm{~mm}$. longo et lato, 
limbo truncato-sublobulato, petalis 4 orbiculato-ovato, ante anthesin $3 \mathrm{~mm}$. longis. Stamina 8 aequalia, filamentis $1 \mathrm{~mm}$. longis latiusculis, antheris late ovatis acutis $1.5 \mathrm{~mm}$. longis, a latere plus minusve compressis, ovario 2-3-loculari depresso glabro, stylo glabro, stigmate capitato, placentis latis planis, seminibus lanceolatis, testa utrinque producta, cum testa $2-3 \mathrm{~mm}$. longis. Ab A. Beccariana Cogn. differt, foliis minoribus, floribus 4-meris nec 5-meris, calyce dense lepidoto.

No. 13911 Kanehira-Hatusima Angi, Arfak Mts., in fringing forests by Iray, Lake Giji, alt. about $1900 \mathrm{~m}$. above the sea, April 8, 1940.

34. Kibessia galeata Cogn. in DC. Monogr. Phanerog. 7 (1891) 1110. var. latifolia OHwI, var. nov.

Arbuscula glaberrima $8 \mathrm{~m}$. alta (ex coll.). Rami teretes brunnei tenues satis ramosi. Folia ovata vel late ovata $10-15 \mathrm{~cm}$. longa $6-8 \mathrm{~cm}$. lata tenuiter coriacea triplinervia, supra viridia, subtus pallidiora (vel glaucescentia?), nervis supra leviter impressis, subtus elevatis, nervis transversalibus (subirregularibus) nervulisque anastomosantibus praesertim subtus prominulis notata, apice breviter acuminata, basi vix vel leviter inaequilatera rotundata vel obtusa, petiolis $3-5 \mathrm{~mm}$. longis a dorso subcompressis vix sulcatis. Flores axillares solitarii, pedunculis $2-3 \mathrm{~mm}$. longis, medio bibracteolatis, bracteolis vix $1 \mathrm{~mm}$. longis deltoideis acutis, basi latissima connatis, stylo persistente $10 \mathrm{~mm}$. longo glabro, stigmate 4-lobo. Capsula subglobosa $8-9 \mathrm{~mm}$. diametiens, supra medium usque tuberculis planis angulatis humillimis vel vix elevatis $1-1.5 \mathrm{~mm}$. latis verrucosa, apice calycis limbo conico demum profunde 4-fido (lobis deltoideis obtusulis $2 \mathrm{~mm}$. longis leviter incurvis) coronata, 4-loculari, placentis basin versus loculorum parietalibus, seminibus cuneatis vix $1 \mathrm{~mm}$. longis angulatis nitidis, laevibus, apice truncatis, hilo minuto basilari, raphe nulla. A typo foliis latioribus basi saepe rotundatis, stigmate vix capitato-4-lobo.

No. 13146 Kanehira-Hatusima, Waren, 60 miles south of Manokwari, in strand forests on rocky slopes, March 26, 1940. Fruit white.

Distr. Sp. Borneo. Another variety in the North-eastern part of New Guinea.

35. Pternandra caerulescens JAck. in Mal. Misc. 2 (1822) 61. var. cyanea (Blume) Cogn. in DC. Monogr. Phanerog. 7 (1891) 1104.

Ewickia cyanea BuUme in Flora (1831) 525 et in Rumphia 1, 24, t. 8.

No. 13109 Kanehira-Hatusima, Waren, 60 miles south of Manokwari, in strand forests on rocky slopes, about $3 \mathrm{~m}$. alt. above the sea, March 26, 1940. Flores subsolitarii. 
36. Memecylon longifolium Cogn. in DC. Monogr. Phanerog. 7 (1891) 1150.

A descriptione paullo diversum: ramis subgracilibus, petiolis 10-15 $\mathrm{mm}$. longis. Foliorum laminae siccitate brunneo-virides, nervis marginalibus flexuosis a marginibus $3-5 \mathrm{~mm}$. distantibus, cum nervulisque transversalibus (pluribus) subtus tantum elevatulis, costa media supra sulcata subtus elevata notata, petiolis supra vix sulcatis, cymae axillares solitariae pauciflorae, pedunculis brevissimis, pedicellis $5-7 \mathrm{~mm}$. longis, bacca ovoidea $10 \mathrm{~mm}$. longa $8 \mathrm{~mm}$. crassa obsolete striata, apice calycis limbo $2-2.5 \mathrm{~mm}$. lato $1 / 2 \mathrm{~mm}$. alto truncato-erosulo terminata, stylo persistente tenui.

No. 12515 Kanehira-Hatusima, Sennen, $40 \mathrm{~km}$. inward of Nabire, in Agathis-forests, alt. about $400 \mathrm{~m}$. above the sea, March 7, 1940. A tree about $4 \mathrm{~m}$. tall, fruits red.

Distr. New Guinea and Borneo.

37. Memecylon sepicanum Mansf. in EngL. Bot. Jahrb. 60 (1926) 141.

Specimen fructiferum, a descriptione diversum tantum pedicellis ca. 5-6 $\mathrm{mm}$. longis, fructibus in siceo olivaceo-viridibus.

No. 11594 K $\Lambda$ Nehira-Hatusima, Nabire, Geelvink Bay, in high rainforests, alt. about $3 \mathrm{~m}$. above the sea, Febr. 26, 1940. A tree about $10 \mathrm{~m}$. high, fruits red.

Distr. Endemic.

38. Memecylon dallmannense OHwi, sp. nov.

?Memecylon hepaticum var. grandifolium Cogn. in DC. Monogr. Phanerog. 7 (1891) 1151.

Rami glabri cinereo-brunnei satis tenues ramosi. Folia cum petiolo 5-10 mm. longo $15-20 \mathrm{~cm}$. longa 5-7 cm. lata tenuiter coriacea oblongo-ovata nitidula, supra obscuriter rubro-fusca, subtus rufescentia, remote penninervia, costis nervisque supra sulcatis, subtus elevatulis, nervulis transversalibus supra leviter, subtus non visibilibus notata, apice acuta usque breviter caudato-acuminata, basi acuta, petiolis supra sulcatis. Cymae axillares fasciculatae subracemoso-dispositae sub-5-florae ca. $3 \mathrm{~cm}$. longae, pedicellis 5-8 mm. longis satis crassis angulatis verruculosis, brevissime puberulis, vel glabrescentibus, apice bracteolatis. Fructus oblique obovoideus $10 \mathrm{~mm}$. longus glaber, apice calycis limbo $1.5 \mathrm{~mm}$. longo sursum aperto leviter 4-lobo (lobis valde depressis) coronatus, basi angustato-truncatus.

No. 12281 Kanehira-Hatusima, Dallmann, Nabire, in Agathis-forests, alt. about $500 \mathrm{~m}$. above the sea, March 3, 1940. A small tree about $3 \mathrm{~m}$. tall. 


\title{
金平・初島探集ニニーギニヤ植物研究 XVI
}

\author{
大 井 次 三 郎
}

のぼれん科

本邦産のぼそん科ノ植物ハ決シテ濢山ノ種類ガアル譯デハナイガ, 先年筆者ガ臺 灣デ探集旅行シ夕際二, 最モ心ヨ引カレタモノノーツデ，此科全體トシテノ美シイ

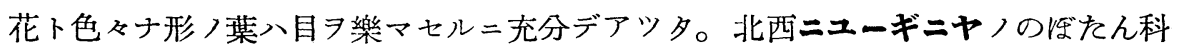
ノ採集品ヨココロヨク貸與サレタ金平・初島兩氏二厚ク謝意ヨ萃スル。

本邦卜共通ノ種類入ーツモナク，Otanthera, Melastoma, Medinilla, Astronia ， 四屬八臺灣及ビソレ 以北二モアルガ Poikilogyne, Sonerila, Phyllapophysis, Dissochaeta, Astronidium, Kibessia, Pternandra, Memecylon 〉諸屬八本邦二ハ未知デア

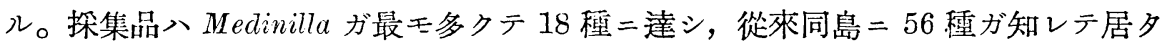
ガ, 此所デ 11 種ガ附加サレタ。ソレニ次イデハAstronia, Memecylon, Poikilogyne ガ各 3 種ヅツアリ，殘リ八各一種ヅツデアツタ。 